\title{
Host Genomics and Control of Tuberculosis Infection
}

\author{
A. Cobat ${ }^{a, b}$ M. Orlova ${ }^{a} \quad$ L.F. Barrerad, e $\quad$ E. Schurr ${ }^{a-c}$ \\ ${ }^{a}$ McGill Centre for the Study of Host Resistance, The Research Institute of the McGill University Health Centre, \\ and Departments of ${ }^{\mathrm{b}}$ Human Genetics and ${ }^{\mathrm{C}}$ Medicine, McGill University, Montreal, Que., Canada; ${ }^{\mathrm{d}}$ Grupo de

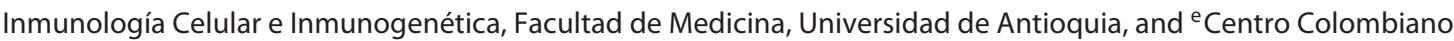 \\ de Investigación en Tuberculosis, Medellín, Colombia
}

\section{Key Words}

Complex traits - Host genetics - Host resistance ·

Latent tuberculosis infection - Mycobacterial diseases .

Tuberculosis gether, these data suggest TB surveillance and control can likely be improved by including host genetic information into the interpretation of these widely used assays.

Copyright $\odot 2013$ S. Karger AG, Basel

\section{Introduction}

Tuberculosis (TB) is an infectious disease caused by the human pathogenic bacterium Mycobacterium tuberculosis. TB is a major health care problem with an estimated 2 billion individuals infected with $M$. tuberculosis world-wide. The global TB incidence in 2010, accounting for new and recurrent cases, has been estimated by the World Health Organization (WHO) at 8.8 million with a death toll of close to 1.5 million. For decades, the highest TB rates have been observed in Asia and Africa, accounting for over $80 \%$ of globally recorded cases. WHO identified 22 countries with high TB endemicity in 2 of which prevalence rates exceeded $1 \%$ of the overall population (South Africa, Cambodia). The economic situation of low- and middle-income countries was and continues to be a substantial impediment for effective TB

\section{A. Cobat and M. Orlova contributed equally to this work.}

\begin{tabular}{ll}
\hline KARGER & $\begin{array}{l}\text { Co } 2013 \text { S. Karger AG, Basel } \\
\text { 1662-4246/13/0162-0044 } \$ 38.00 / 0 \quad \text { Kar }\end{array}$ \\
E-Mail karger@karger.com & $\begin{array}{l}\text { This is an Open Access article licensed under the terms of } \\
\text { the Creative Commons Attribution-NonCommercial-No- } \\
\text { www.karger.com/phg }\end{array}$ \\
& $\begin{array}{l}\text { Derivs 3.0 License (www.karger.com/OA-license), applica- } \\
\text { ble to the online version of the article only. Distribution for } \\
\text { non-commercial purposes only. }\end{array}$
\end{tabular}

Erwin Schurr

Montreal General Hospital Research Institute, Room L11-521 1650 Cedar Avenue

Montreal, QC H3G 1A4 (Canada)

E-Mail erwin.schurr@ mcgill.ca 
Fig. 1. Schematic representation of TB pathogenesis. From exposure to $M$. tuberculosis to progression to active TB disease. The different stages of pathogenesis are influenced by a variety of environmental (microbial/non-microbial) and host (genetic/ non-genetic) factors.

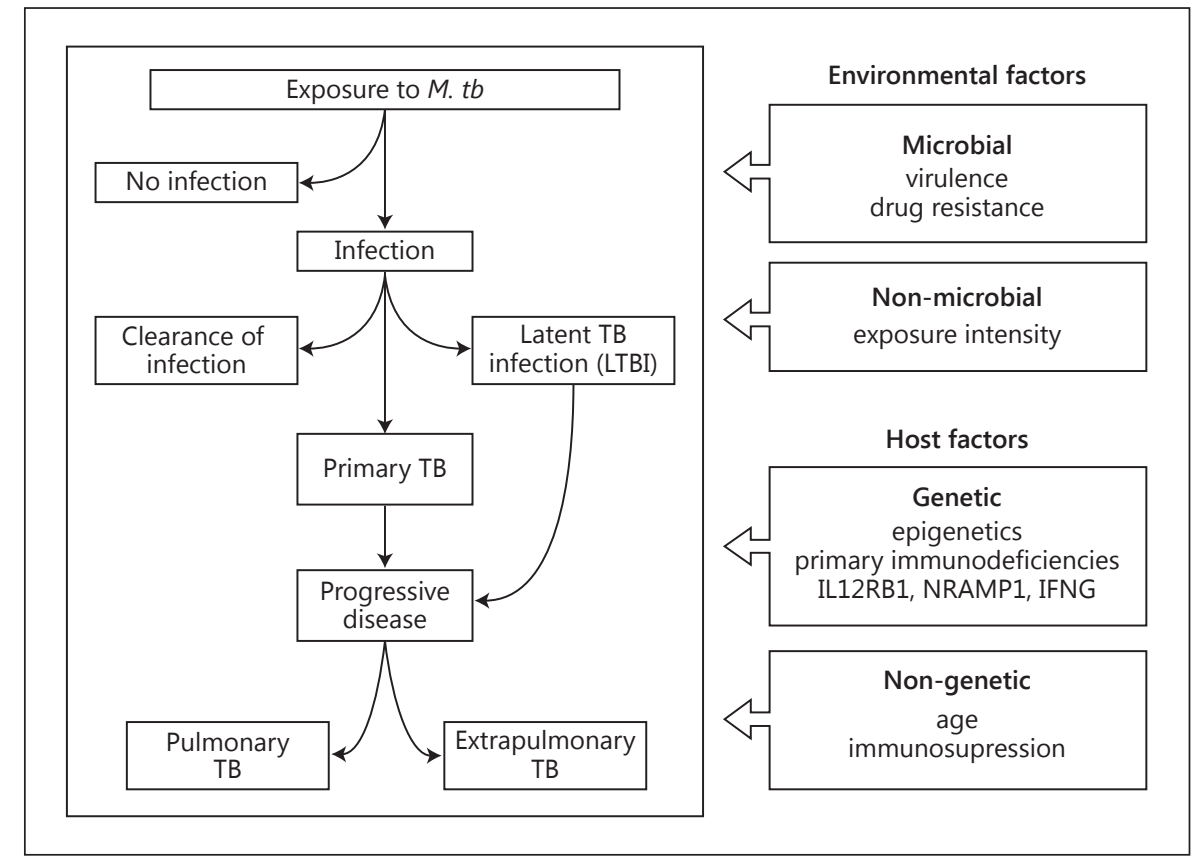

control and treatment programs. The occurrence and spread of multi-drug- and extremely drug-resistant (MDR/XDR) strains of M. tuberculosis combined with the HIV epidemic has added substantial problems and challenges to global TB control programs. By contrast, wealthier nations such as North American and Western European countries contribute only a small proportion of the world TB burden ( 5\%), with incidence rates less than 5 cases per 100,000. In countries of low endemicity, TB is predominantly recorded among immigrants from high-prevalence countries, immuno-compromised individuals (e.g. elderly, persons with HIV/AIDS), homeless people, and other economically disadvantaged groups. Due to different epidemiological situations, TB control programs in low-incidence regions target different aspects of TB pathogenesis than those in high-incidence settings.

\section{TB: The Pathogenesis}

Transmission of $M$. tuberculosis bacilli occurs through aerosolized droplets expelled by coughing cases with active disease that are being inhaled by contacts of patients with active TB disease (fig. 1). There is a pronounced inter-individual variability of response to $M$. tuberculosis exposure. Based on TB case contact studies, approximately half of exposed persons clear the bacilli before develop- ment of persistent infection. In those persons where successful infection occurs, only an estimated $5 \%$ will develop clinical disease within 1-2 years of infection while in the remaining 95\% the immune system reaches a state of equilibrium with the M. tuberculosis pathogen. Individuals with such latent TB infection (LTBI) display no symptoms of disease and are non-infectious. Due to their asymptomatic presentation, the majority of LTBI cases in high incidence regions remain undiagnosed, thereby providing a large reservoir for future $M$. tuberculosis transmission. Indeed, an estimated 5\% of healthy LTBI persons will lose control of their latent infection and develop TB during their life-time. In immune-suppressed persons, including AIDS patients, the annual risk of TB is hugely increased and may reach $10 \%$ per year (fig. 1).

\section{TB Disease: The Diagnosis and Its Challenges}

The priority given to identify cases with LTBI varies between countries depending on TB burden. While highincidence countries mainly focus on treating notified and self-reporting active cases, well-developed countries with low TB endemicity put significant effort into identification and treatment of LTBI cases to minimize further transmission. These different approaches highlight the fact that eventual elimination of TB as public health threat will not be possible without transmission-blocking inter- 
ventions, i.e. the prevention of new infections, and the eradication of the LTBI reservoir of $M$. tuberculosis.

Until recently, TB diagnostic methods used for the past century had remained almost unchanged and were based on the identification of $M$. tuberculosis by microscopy or culture. This presented significant limitations and challenges for an accurate, timely diagnosis. However, substantial improvement for point-of-care diagnosis has been made with the introduction of the GeneXpert MTB/RIF gene amplification system. The Xpert MTB/ RIF is a rapid, closed automated system that employs the real-time polymerase chain reaction to detect $M$. tuberculosis in sputum while concurrently testing for rifampicin resistance of the patient isolate (reviewed in [1]). Widespread use of the system in endemic countries is hampered by the high cost of the assay and by its current inability to classify clinical isolates into MDR/XDR $M$. tuberculosis strains. Given their increasing prevalence point-of-care tests for MDR/XDR strains are one of the grand challenges of TB control.

\section{Latent Infection: The Diagnosis and Its Challenges}

Until recently, the only available test used to identify LTBI was the tuberculin skin test (TST). Developed over a century ago, TST detects cell-mediated immunity in the form of a delayed type hypersensitivity response to purified protein derivative (PPD) injected intradermally in the patient's forearm. PPD presents a mixture of protein antigens, derived from heated $M$. tuberculosis cultures, which may overlap with proteins from $M$. bovis (including Bacillus Calmette-Guerin (BCG) vaccine strains) and a number of environmental non-tuberculous mycobacteria (NTM) [2]. Due to the overlap of antigens, test results measured as skin induration after $48-72 \mathrm{~h}$ at the site of injection may show reduced specificity after exposure to NTM or in populations with obligatory BCG vaccination. In low-incidence countries, TST testing is principally targeted at persons with high risk of reactivation: recent immigrants from high-burden countries, HIV-infected patients and organ recipients, patients under cancer or long-term systemic corticosteroid treatments, and patients eligible for TNF blocker therapy. In high TB-burden countries with an ongoing HIV epidemic, WHO adopted a policy of TST screening for latent TB, as it remains among the leading causes of mortality for HIVinfected patients (reviewed in [1]). Trained and experienced personnel are required for administration and reading of the test for which the patient has to be seen in
2-3 days after initial PPD injection. Despite such limitations, the TST is widely endorsed due to low cost and ease of use outside of laboratory settings, and there is a large knowledge base regards the interpretation of TST results in a wide array of epidemiological and clinical settings.

Interferon- $\gamma($ IFN- $\gamma)$ release assays (IGRAs) were designed to replace the TST due to some of the perceived shortcomings listed above. Like the TST, these tests are based on detection of $M$. tuberculosis-specific cell-mediated immunity in LTBI persons. Stimulation of M. tuberculosis-specific T-cells by M. tuberculosis test antigens elicits release of various signaling cytokines including IFN- $\gamma$. Higher M. tuberculosis detection specificity of IGRAs is accomplished through utilization of M. tuberculosis-specific challenge antigens encoded by the genomic region of difference 1 absent in BCG vaccine strains and most NTMs. Two commercially available kits, QuantiFERON-TB Gold In Tube (QFT-GIT, Cellestis, Australia) and T-SPOT-TB (Oxford Immunotec, UK), are commonly used (reviewed in [3]). For the QFT-GIT kit, blood is incubated with M. tuberculosis antigens (as well as positive and negative controls) for approximately $20 \mathrm{~h}$ at which time the amount of IFN- $\gamma$ secreted into the plasma is determined by enzyme-linked immunosorbent assay. The differential production of antigen stimulated versus background IFN- $\gamma$ indicates if a patient is presently infected by $M$. tuberculosis. The QFT-GIT method may give indeterminate results, especially if the mononuclear cell count in blood is low. Compared to the QFTGIT method, the T-SPOT-TB is more laborious as it requires blood fractionation followed by a more costly enzyme-linked immunospot IFN- $\gamma$ release assay. However, due to the cell separation and concentration step, the TSPOT-TB does provide very accurate results.

In low-incidence settings, IGRAs compared to TST have higher specificity and less cross-reactivity with previous BCG vaccination and exposure to NTM. Sensitivity of IGRAs, however, is variable and not always higher than that of TST, especially in high-risk populations. Valid IGRAs results are usually obtained within 1-2 days after a single patient visit. Similar to TST, IGRA results are prone to interpretation when different considerations are taken into account (i.e. coinfections, exposure to active $\mathrm{TB}$, age, pregnancy). Unlike TST, IGRAs are expensive and call for well-equipped laboratory setting and highly trained personnel, making it more feasible for diagnostic practice in low-burden, high-income countries. However, it is clear that the in vitro IGRAs and the in vivo TST measure different aspects of the anti-mycobacterial immune response. In the absence of a gold standard for di- 
agnosis of LTBI, the most judicial approach is to use both IGRA and the TST assays to arrive at a valid diagnosis of LTBI.

\section{Human Genetic Factors of LTBI}

Recent molecular studies have provided proof-ofprinciple that susceptibility to tuberculosis disease can result from genetic predisposition with the identification of children with Mendelian predisposition to disseminated tuberculosis [4]. Moreover, identification of the genetic factors predisposing to pulmonary $\mathrm{TB}$ disease is the subject of intense research activities. A major locus on chromosomal region $8 \mathrm{q} 12-13$ for pulmonary TB has been identified in a Moroccan population by genome-wide linkage analysis [5] and numerous genes and pathways have been implicated by candidate gene association studies (reviewed in [6]). More recently, a genome-wide association study followed by imputation of data from the 1000 Genomes Project identified common variants with a modest effect size on pulmonary TB disease at chromosome regions 18q12 [7] and 11p13 [8] in an African population. However, the success in identifying host genetic factors that impact on risk of clinical TB disease in these studies has been quite limited. Experimental evidence from both animal models and human studies has emerged suggesting that host genetic control of TB involves specificity with respect to the mycobacterial strain encountered [9]. In addition, the accidental inoculation of infants with variable doses of $M$. tuberculosis during the so-called Lübeck accident had shown that exposure intensity is a critical factor in the etiology of primary TB, i.e. TB within 2 years of infection. These initial observations have been confirmed by more recent molecular studies that showed a strong impact of crudely modeled exposure histories on the ability to detect strong genetic risk factors of primary TB [10]. To tackle the emerging host gene environment interactions is a major challenge for the study of the host genetic control of clinical TB disease.

Despite the fact that LTBI represents a huge reservoir of $M$. tuberculosis contributing to the 'success' of the pathogen with an estimated one-third of the world population infected by M. tuberculosis on the basis of the TST, few studies have aimed to dissect its genetic basis. Only recently have studies emerged that focused on TST and/ or IGRA reactivity as quantitative or binary traits, and those studies have consistently provided strong evidence for an important role of human genetics in LTBI. Several family studies have reported high levels of heritability (i.e. proportion of the phenotypic variance explained by an additive genetic component where the effect of 2 responder alleles is double of carrying a single responder allele) for both TST and IGRA responses in the context of M. tuberculosis exposure [11-13]. In Chile, the sib-sib correlation of quantitative TST reactivity (in $\mathrm{mm}$ ) was estimated at 0.46 among young, healthy children exposed to an active TB case [13]. Heritability of TST reactivity could thus be estimated at $92 \%$ (i.e. twice the sib-sib correlation coefficient) in this study. In The Gambia, an endemic country for TB disease, a study of healthy twins aged 12-83 years estimated the heritability of TST considered as a categorical trait and quantitative IGRA reactivity at 71 and $39 \%$, respectively [12]. In a familial sample composed of healthy children and young adults from a hyper-endemic area for TB disease, we estimated the heritability of the quantitative IGRA response between 43 and $58 \%$, depending on the nature of stimulating antigen [11]. Only one family study conducted in Uganda reported a low heritability estimate for IGRA ( 17\%) [14]. However, in this study, IGRA reactivity had been adjusted on TST response prior to the analysis, thus removing the shared genetic component between TST and IGRA. It is likely that this lead to an underestimation of the true IGRA heritability. Given the high heritability of TST and IGRA, there must be human genes that play an important role in tuberculosis infection and the establishment of LTBI. In the following sections, we will focus on TST reactivity, which has been studied more intensely than the IGRAs.

Recently, we performed the first complex segregation analysis of TST reactivity in related household contacts of tuberculosis index cases in a Colombian population [15]. In general terms, the aim of complex segregation analyses is to determine whether the observed familial correlations of the trait of interest are compatible with the transmission from parents to offspring of a major gene and to estimate the characteristics of this gene. We detected evidence for a major co-dominant gene explaining $\sim 65 \%$ of TST variability in this Colombian population [15]. The frequency of the allele predisposing to a low TST value was estimated at 0.56 . Such a low responder allele frequency meant that nearly one-third of the study population was predisposed to low TST reactivity (with an estimated TST genotypic mean of $3.4 \mathrm{~mm}$ ). Conversely, a high responder allele frequency of 0.44 implied that approximately $20 \%$ of the population was predisposed to high TST reactivity (with an estimated TST genotypic mean of $20.9 \mathrm{~mm}$ ). The results obtained were in agreement with the high estimates of TST heritability reported 
in the previous studies $[12,13]$ and suggested that TST is a promising phenotype for subsequent molecular genetics studies.

Only few molecular studies have been conducted so far to identify the genetic factors underlying LTBI susceptibility and TST reactivity. Candidate gene association studies have focused on TST response as a binary trait according to various thresholds $(0,5$ or $10 \mathrm{~mm})$ [16-18]. In Ghana, an IL10 promoter haplotype (-2849G/-1082G/$819 \mathrm{C} /-592 \mathrm{C}$ ) associated with high levels of circulating IL10 was significantly more frequent in TST non-responders (cutoff not specified) compared to TST responders (15.3 vs. $9.7 \%, \mathrm{OR}=2.09, \mathrm{p}=0.01$ ) [18]. Interestingly, the prevalence of TST non-response was found 1.5 times higher among individuals carrying the genotype GG at SNP $-1082 \mathrm{~A}>\mathrm{G}$ compared to individuals carrying the AA and $A G$ genotypes in an indigenous population from Brazil [16]. The later study also reported significant association of TST response with SNPs in IL4 $(-590 \mathrm{~T}>\mathrm{C}, \mathrm{p}=$ $0.007)$ and $I F N-\gamma(+874 \mathrm{~A}>\mathrm{T}, \mathrm{p}=0.02)$ genes, but the results need to be replicated in independent studies.

In candidate gene studies, only 'relevant' genes based on the a priori knowledge of their biological function are investigated, thus precluding the identification of new genes or pathways involved in the phenotype of interest. To better characterize the genetic architecture of LTBI, 2 studies performed genome-wide linkage screens of TST reactivity considered either as a binary $[19,20]$ or a quantitative trait [19]. In Uganda, a genome-wide linkage analysis reported suggestive, but not significant, linkage of persistent TST negativity (defined as a TST $<10 \mathrm{~mm}$ or $5 \mathrm{~mm}$ according to age and HIV status) with chromosomal regions 2q21-2q24 and 5p13-5q22 [20]. By focusing on TST negativity per se, (0 mm vs. non 0$)$ in Cape Town, South Africa, a major locus, coined TST1 ( $\mathrm{p}=$ $10^{-5}$ ), on chromosomal region $11 \mathrm{p} 14$ was identified [19]. The same study identified a major locus, coined TST2 ( $\mathrm{p}$ $<10^{-5}$ ), on chromosomal region $5 \mathrm{p} 15$ that controls the extent of TST reactivity. Preliminary fine-mapping of the TST2 locus led to the identification of SLC6A3, a gene involved in dopamine transport in humans and the control of delayed type hypersensitivity in mice, as an interesting candidate gene to control the extent of the TST reaction.

\section{Conclusion}

Several exciting avenues of research emerge from the above discussed results. From a public health perspective, any parameter that impacts on commonly used measures of TB surveillance is interesting. In this regard, the above studies provide clear and unambiguous evidence for a strong impact of the host genetic background on TST and suggest that the underlying genetic variants can be molecularly identified. Once that has happened, the integration of host genetic variation into TB disease surveillance will become a new reality and demonstrate the usefulness of modern genomics for the control of infectious diseases.

\section{References}

1 McNerney R, Maeurer M, Abubakar I, Marais B, McHugh TD, Ford N, Weyer K, Lawn S, Grobusch MP, Memish Z, Squire SB, Pantaleo G, Chakaya J, Casenghi M, Migliori GB, Mwaba P, Zijenah L, Hoelscher M, Cox H, Swaminathan S, Kim P, Schito M, Harari A, Bates M, Schwank S, O'Grady J, Pletschette M, Ditui L, Atun R, Zumla A: Tuberculosis diagnostics and biomarkers: needs, challenges, recent advances, and opportunities. J Infect Dis 2012; 205(suppl 2):S147-S158.

$>2$ Affronti LF, Caprio JJ, Edwards PQ, Furculow ML, Grzybowski S, Katz J, Hesse FE, Seibert FB: What is PPD-S? A statement by the Committee on Diagnostic Skin Testing. Am Rev Respir Dis 1969;99:460-461.
3 Pai M, Joshi R, Kalantri S: Diagnosis of latent tuberculosis infection: recent advantages and future directions; in Sharma SK, Mohan A (eds): Tuberculosis. New Delhi, Jaypee Brothers Medical Publishers (P) Ltd., 2009, pp 186199.

4 Alcaïs A, Fieschi C, Abel L, Casanova JL: Tuberculosis in children and adults: two distinct genetic diseases. J Exp Med 2005;202:16171621.

5 Baghdadi JE, Orlova M, Alter A, Ranque B, Chentoufi M, Lazrak F, Archane MI, Casanova JL, Benslimane A, Schurr E, Abel L: An autosomal dominant major gene confers predisposition to pulmonary tuberculosis in adults. J Exp Med 2006;203:1679-1684.

6 Möller M, Hoal EG: Current findings, challenges and novel approaches in human genetic susceptibility to tuberculosis. Tuberculosis (Edinb) 2010;90:71-83.
48

Public Health Genomics 2013;16:44-49 DOI: $10.1159 / 000341499$
Cobat/Orlova/Barrera/Schurr
7 Thye T, Vannberg FO, Wong SH, Owusu-Dabo E, Osei I, Gyapong J, Sirugo G, Sisay-Joof F, Enimil A, Chinbuah MA, Floyd S, Warndorff DK, Sichali L, Malema S, Crampin AC, Ngwira B, Teo YY, Small K, Rockett K, Kwiatkowski D, Fine PE, Hill PC, Newport M, Lienhardt C, Adegbola RA, Corrah T, Ziegler A; African TB Genetics Consortium; Wellcome Trust Case Control Consortium, Morris AP, Meyer CG, Horstmann RD, Hill AV: Genome-wide association analyses identifies a susceptibility locus for tuberculosis on chromosome 18q11.2. Nat Genet 2010;42:739741 
-8 Thye T, Owusu-Dabo E, Vannberg FO, van Crevel R, Curtis J, Sahiratmadja E, Balabanova Y, Ehmen C, Muntau B, Ruge G, Sievertsen J, Gyapong J, Nikolayevskyy V, Hill PC, Sirugo G, Drobniewski F, Van De Vosse E, Newport M, Alisjahbana B, Nejentsev S, Ottenhoff TH, Hill AV, Horstmann RD, Meyer CG: Common variants at $11 \mathrm{p} 13$ are associated with susceptibility to tuberculosis. Nat Genet 2012;44:257-259.

$\checkmark 9$ Di Pietrantonio T, Hernandez C, Girard M, Verville A, Orlova M, Belley A, Behr MA, Loredo-Osti JC, Schurr E: Strain-specific differences in the genetic control of two closely related mycobacteria. PLoS Pathog 2010; 6:e1001169.

10 Schurr E: Is susceptibility to tuberculosis acquired or inherited? J Intern Med 2007;261: 106-111.

$\checkmark 11$ Cobat A, Gallant CJ, Simkin L, Black GF, Stanley K, Hughes J, Doherty TM, Hanekom WA, Eley B, Beyers N, Jais JP, van Helden P, Abel L, Hoal EG, Alcais A, Schurr E: High heritability of anti-mycobacterial immunity in an area of hyperendemicity for tuberculosis disease. J Infect Dis 2010;201:15-19.
12 Jepson A, Fowler A, Banya W, Singh M, Bennett $S$, Whittle $H$, Hill AV: Genetic regulation of acquired immune responses to antigens of Mycobacterium tuberculosis: a study of twins in West Africa. Infect Immun 2001;69:39893994.

13 Sepulveda RL, Heiba IM, King A, Gonzalez B, Elston RC, Sorensen RU: Evaluation of tuberculin reactivity in BCG-immunized siblings. Am J Respir Crit Care Med 1994;149:620 624.

14 Stein CM, Guwatudde D, Nakakeeto M, Peters P, Elston RC, Tiwari HK, Mugerwa R, Whalen CC: Heritability analysis of cytokines as intermediate phenotypes of tuberculosis. J Infect Dis 2003;187:1679-1685.

15 Cobat A, Barrera LF, Henao H, Arbeláez P, Abel L, García LF, Schurr E, Alcaïs A: Tuberculin skin test reactivity is dependent on host genetic background in Colombian tuberculosis household contacts. Clin Infect Dis 2012; 54:968-971.

16 Zembrzuski VM, Basta PC, Callegari-Jacques SM, Santos RV, Coimbra CE, Salzano FM, Hutz MH: Cytokine genes are associated with tuberculin skin test response in a native Brazilian population. Tuberculosis (Edinb) 2010; 90:44-49.
17 Gómez LM, Anaya JM, Sierra-Filardi E, Cadena J, Corbí A, Martín J: Analysis of DCSIGN (CD209) functional variants in patients with tuberculosis. Hum Immunol 2006;67: 808-811.

18 Thye T, Browne EN, Chinbuah MA, Gyapong J, Osei I, Owusu-Dabo E, Brattig NW, Niemann S, Rüsch-Gerdes S, Horstmann RD, Meyer CG: IL10 haplotype associated with tuberculin skin test response but not with pulmonary TB. PLoS One 2009;4:e5420.

19 Cobat A, Gallant CJ, Simkin L, Black GF, Stanley K, Hughes J, Doherty TM, Hanekom WA, Eley B, Jaïs JP, Boland-Auge A, van Helden P, Casanova JL, Abel L, Hoal EG, Schurr E, Alcaïs A: Two loci control tuberculin skin test reactivity in an area hyperendemic for tuberculosis. J Exp Med 2009;206:2583-2591.

20 Stein CM, Zalwango S, Malone LL, Won S, Mayanja-Kizza H, Mugerwa RD, Leontiev DV, Thompson CL, Cartier KC, Elston RC, Iyengar SK, Boom WH, Whalen CC: Genome scan of $M$. tuberculosis infection and disease in Ugandans. PLoS One 2008;3:e4094. 